\title{
Uniform Transient Error ${ }^{1}$
}

\author{
Edith L. R. Corliss
}

\begin{abstract}
The economy of transient error is discussed. Equations describing error in powerlevel measurements of transients can be used to compute the design of analyzers so as to distribute transient error in a way compatible with experimental requirements. In addition, consideration of a limiting power-discrimination factor provides a measure of the largest number of bandpass filters that can be overlapped on adjacent channels to yield meaningful information about a rapidly changing signal. A scanning filter can be compared with a set of bandpass filters by calculating the effective number of overlapped bandpass filters providing the same resolution as the scanning filter. The results are applicable to autocorrelation analyzers. Restrictions on rapid signal analysis also are considered for the special case in which power-level discrimination is limited by noise.
\end{abstract}

\section{Introduction}

Measuring systems are limited by natural laws, and several of these guarantee that errors are inevitable. However, the distribution of errors can be controlled by the experimenter. One can choose experimental conditions that minimize errors in a region of particular interest; one can choose alternatively to spread the errors out as thinly as possible.

The transient properties of a measuring system become dominant whenever there is a restriction in time. The constraint may arise either because a signal is to be analyzed by a rapid process or because a signal may be changing rapidly in time.

In order to minimize transient error, experimental conditions must be chosen on a quantitative basis. Some prior knowledge of the signal to be analyzed is required. For the purpose of choosing the conditions of measurement, the problem of analysis can be restated as follows: Given an ensemble of signals characterized by a range of fluctuations in power over a range of intervals in time, one certain signal is to be distinguished from all other signals in the group.

A signal is discrete in time. The fluctuations in power during the existence of the signal convey its identity. If the power-level fluctuations are to be indicated within a certain degree of error, the time required for the analyzer to build up to within a corresponding fraction of its steady-state value must not exceed the shortest time interval over which a significant fluctuation of power level occurs in the component of the signal being analyzed. Thus, the rate at which the indication can build up or decay in the analyzer must exceed a limiting value. It is useful to describe this rate by reference to the response time of the analyzer, i. e., the time required to reach substantially steady-state indication after an abrupt change in signal.

Regardless of whether a frequency or a timecorrelation analysis of the signal is undertaken, the results are observed as output power levels. Hence the ability to resolve a change in power level limits the discrimination that can be achieved for changes in frequency or intervals of time. The relation be-

1 This work was carried out as part of a program of pasic instrumentation research and development cooperatively supported by Office of Naval Research, Atomic Energy Commission, Air Force Office of Scientific Research, and the National Bureau of Standards. tween the various limitations on the performance of an analyzer can be illustrated by means of a threedimensional system (see fig. 1) in which the Cartesian coordinates are frequency, time, and either power or a function of power. The transient error then becomes a least volume in this space, within which no information about the signal can be found. The size of this least volume is limited by the natural behavior of analyzers, but its shape can be distorted to suit the needs of the experimenter.

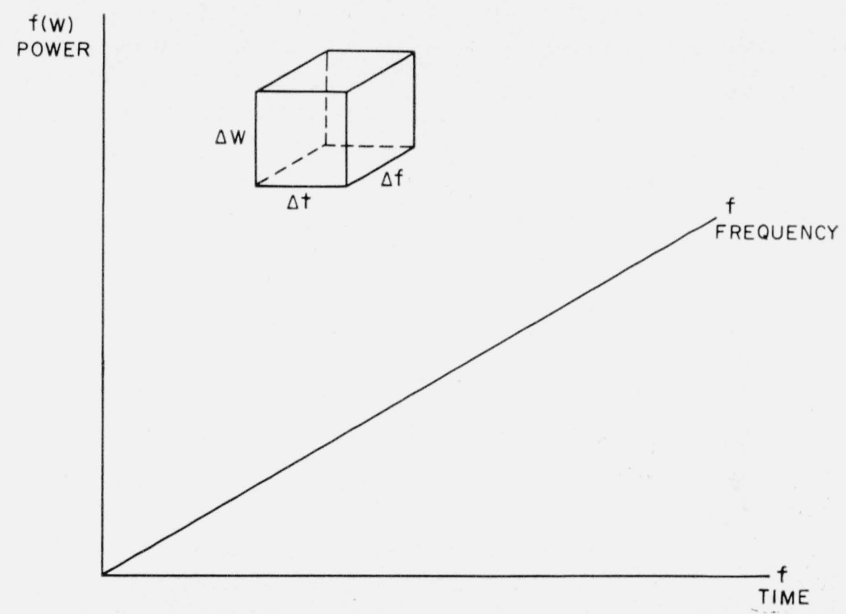

Figure 1. Three-dimensional signal space.

Resolutions in frequency and time are implicit in the $Q$ (figure of merit) ${ }^{2}$ of a frequency analyzer or, correspondingly, in the integration time of a correlation analyzer. To minimize the errors in an analysis, either $Q$ or the integration time must be kept as high as possible consistent with the necessary resolution in power level.

Consider the rate at which power builds up in a linear series-resonant system, in a simple filter or a galvanometer. A family of indicial response curves for such a system is shown in figure 2 , in which the coordinates have been scaled to relative values. The amplitude response has been scaled with the steady. state value as a unit; unity on the time scale is the natural, undamped period of the system. Curves in the family are plotted for different values of $Q$.

2 The ratio of the energy-storage capacity of a resonating system to its energy . dissipation rate is known as its "figure of merit", or Q. 


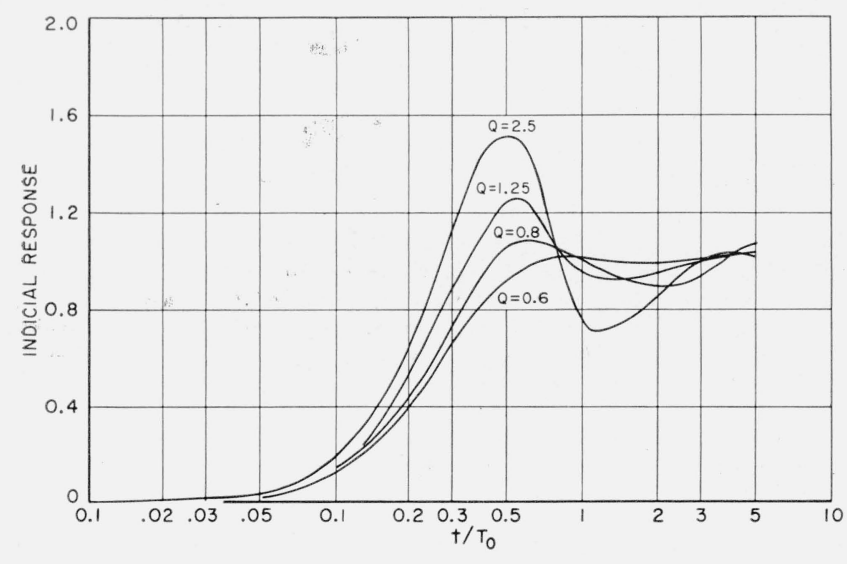

FIGURE 2. Indicial response of a series-resonant system subjected to a step function.

This shows the fraction of steady-state response attained as a function of the time following the impulse, scaled in units of the natural period of the system.

The analyzer may be considered to have reached a sufficient fraction of steady-state indication when it is just capable of resolving all unlike signals, whereas all like signals are indicated as being the same. Attempts either to speed up the response or increase selectivity are obtained at the expense of decreased $Q$ or increased response time, without providing any additional information about the signal. Lengthening the response time, moreover, impairs the ability of the analyzer to indicate rapid power fluctuations, so that some features of the signal can be lost in this way.

\section{Transient Limitations of a Simple Analyzer}

The quantitative extent to which power-level resolution affects resolution along the coordinates of frequency and time can be found from the natural properties of the analyzer. The discussion to follow will be based on the common type of system whose behavior is described by a second-degree differential equation with constant coefficients, but its application to other systems will be indicated.

For example, consider the use of a linear seriesresonant system as a tuned filter to indicate how a particular component of a complex signal fluctuates with time. If $x$ represents amplitude, the transient response of the filter in terms of its center frequency, $f_{0}$, and figure of merit $Q$, is

$$
x=e^{-\pi f_{0} t / Q}\left(x_{1} e^{j 2 \pi \sqrt{f_{0}^{2}-\left(f_{0}^{2} / 4 Q^{2}\right) t}}+x_{2} e^{-j 2 \pi \sqrt{f_{0}^{2}-\left(f_{0}^{2} / 4 Q^{2}\right)} t}\right) .
$$

Let the observation interval, $\Delta \tau$, be sufficiently long compared with $T_{0}$, the undamped natural period of the filter, so that only the envelope of its response need be considered.

The analyzer should be able to change its indication by at least the smallest detectable power increment during a specified time after onset or removal of the input signal. The power built up in the filter is proportional to $|x|^{2}$. If $W_{0}$ is the initial power in the filter, at any time after cessation of the signal the power $W_{t}$ is given by

$$
W_{t}=W_{0} e^{-\frac{2 \pi f_{0}}{Q} t}=W_{0} e^{-\frac{2 \pi}{Q} \frac{t}{T_{0}}}
$$

Let $\Delta W=\left(1-e^{-\alpha}\right) W$ be the smallest discernible change of power level in the filter; i. e., the power must decay by a factor $e^{-\alpha}$ for a change to be observed. If signal cessation is to be discerned within time

$$
W_{0}-W_{\Delta \tau}=W_{0}\left(1-e^{-\frac{2 \pi}{Q} \frac{\Delta \tau}{T_{0}}} \geq\left(1-e^{-\alpha}\right) W_{0},\right.
$$

so that

$$
e^{-\left(\frac{2 \pi}{Q} \frac{\Delta \tau}{T}\right)} \leq e^{-\alpha}
$$

and the requirement is

$$
Q \leq \frac{2 \pi \Delta \tau}{\alpha T_{0}}=\frac{2 \pi f_{0} \Delta \tau}{\alpha}
$$

if the filter is to indicate the occurrence of a change in signal during the interval $\Delta \tau$.

Equation (2) has been derived upon the basis of the finest resolution in power levels that can be achieved with the analyzer in the time interval $\Delta \tau$. Given that $\Delta \tau$ is the briefest duration of any signal component to be observed, eq (2) also shows how the $\mathrm{Q}$ of a filter must be adjusted as a function of its tuning in order to distribute the transient error uniformly over the frequency range. In addition, because eq (2) deals with limits of resolution, it provides a measure of the largest number of bandpass filters that can be overlapped on adjacent channels to yield meaningful information about a rapidly changing signal.

Over a range of frequencies $\Delta f=f_{u}-f_{l}$ surrounding $f_{o}$, the frequency to which the filter is tuned, the change in filter response does not exceed the smallest detectable power change. Consider the relative transmission characteristic of a filter having its $Q$ given by eq (2) (see fig. 3). The relative power response of the filter is given by

$$
W_{f}=\frac{W_{0}}{f^{2} / f_{0}^{2}+Q^{2}\left(1-f^{2} / f_{0}^{2}\right)^{2}} .
$$

The precise result of solving for the discrimination limits in terms of $f_{u}$ and $f_{l}$ comes out as

$$
\frac{f-f_{l}^{2}}{f_{0}^{2}} \geq \frac{\alpha}{\pi f_{0} \Delta \tau} \sqrt{\left(e^{\alpha}-1\right)+\frac{\alpha^{2}}{16 \pi^{2} f_{0}^{2} \Delta \tau^{2}}},
$$

but if $\Delta W$ is small compared to $W_{o}$ (i.e., fine-power discrimination can be made) then

$$
f_{u}-f_{l}=\Delta f \geq \frac{\alpha^{3 / 2}}{2 \pi \Delta \tau} .
$$




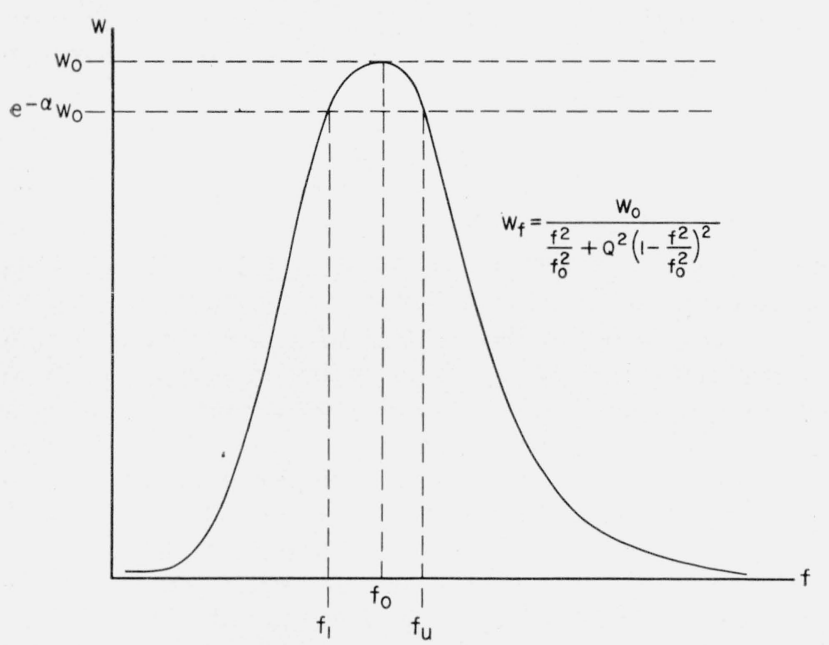

FIGURE 3. Relative power response of a series-resonant system as a function of steady-state driving frequency.

\section{Analyzers Made Up of Overlapped Channels}

For a set of filters overlapped at the limits of the power resolution, the limiting number of filters yielding meaningful information between $f_{t}$ and $f_{b}$, the high-and low-frequency range limits, respectively, is given by

$$
n_{l}-1 \leq \frac{f_{t}-f_{b}}{f_{u}-f_{l}}=\frac{2 \pi \Delta \tau}{\alpha^{3 / 2}}\left(f_{t}-f_{b}\right) .
$$

(The limits yield $n_{l}-1$ rather than $n_{l}$ because one-half of a filter extends beyond each end of the frequency range as defined here.)

In practice, it is usual to overlap adjacent filters at their half-power points, so that their integrated response has a flat characteristic. For this condition, $\Delta f=f_{0} / Q$, and the number of filters becomes

$$
n_{2}-1=\frac{f_{t}-f_{b}}{\Delta f}=\frac{Q\left(f_{t}-f_{b}\right)}{f_{0}}=\frac{2 \pi \Delta \tau\left(f_{t}-f_{b}\right)}{\alpha} .
$$

The ratio of this number to the maximum usable number of filters (overlapped at their limits of resolution) turns out to depend only on power resolution, i.e.,

$$
\frac{n_{2}-1}{n_{l}-1}=\alpha^{1 / 2}
$$

If we are willing to take a "yes-no" type of analysis from the filter, then for a set of filters overlapped at their half-power points we can choose as a compatible condition that $\alpha=\ln 2$, i.e., the power declines at least to one-half its initial value during the observation interval $\Delta \tau$, and we get as the number of filters for this case:

$$
n_{b}-1=\frac{2 \pi \Delta \tau\left(f_{t}-f_{b}\right)}{\ln 2} .
$$

Under these conditions, $n_{b}$ invites comparison with the number of "bits" available in a given bandwidth during the time interval $\Delta \tau .^{3}$

\section{Scanning Analyzers}

The process of scanning makes it possible to study the spectrum of a signal by means of a single channel in place of the numerous channels needed when fixedbandpass filters are operated in parallel. When one scans by sweeping the tuning of a filter over the frequency range to be covered, the filter shows a rather complicated type of response. ${ }^{4}$ However, one can make a quantitative study of the effects of scanning by use of simple theory, if one recognizes that another multiplicative factor will be necessary to take accurate account of the spreading of the filter's transient response due to the effects of scanning. The spreading occurs because the incoming signal beats with the ringing of the filter.

If one sweeps through a band of frequencies included between the limits $f_{t}$ (high-frequency) and $f_{b}$ (low-frequency) in the time interval $\tau$, the length of time available to build up the power response of the filter at any instantaneous tuning is generally much smaller than the whole sweep interval. Call the observation interval for each effective single filter $\Delta \tau$, as for discussion of the fixed-bandpass filter. This quantity is directly related to the length of time that it takes the center tuning of the filter to change through a range of frequencies equal to the bandwidth between its half-power points. In this simplified discussion we shall take this relationship as unity, remembering that it is an approximation.

Various types of scanning functions can be used, depending upon the nature of the selectivity characteristic desired. If one wishes to have a constant $Q$ (constant percentage bandwidth), the condition that the transient error is to be uniform throughout the range of frequencies swept over can be satisfied by scanning at a rate proportional to the square of the instantaneous center tuning of the filter. When one wishes to scan through the frequency range with a filter whose bandwidth is a constant number of cycles per second, so that the $Q$ is directly proportional to the center tuning of the filter, the rate of sweep should be constant independent of frequency. One type of scanning filter sweeps logarithmically over its frequency range; when it is adjusted to uniform transient response, its instantaneous $Q$ is proportional to the square root of its instantaneous tuning. Thus it is intermediate in its properties between constant-frequency bandwidth and constant-percentage bandwidth.

3 R. V. L. Hartley, Transmission of information, Bell System Tech. J. 7, 535 $(\underset{4 \mathrm{~F}}{1928)}$

${ }^{4}$ F. M. Lewis, Vibration during acceleration through a critical speed, Trans. Am. Soc. Mech. Engr. 54, 253 (1932); G. Hok, Response of linear resonant system to excitation of a frequency varying linearly with time, J. Appl. Phy. 19, 24 (1948); N. F. Barber and F. Ursell, The response of a resonant system to a gliding tone, Phil. Mag. 39, 345 (1948); J. Marique, The response of RLC resonant circuit to EMF of sawtooth varying frequency, Proc. Inst. Radio Engrs. 40, 945 (1952) M. C. Herrero, Resonance phenomena in time-varying circuits, Technical Repor No. 69, (Electronics Research Laboratory, Stanford University, 1953); H. W Batten, R. A. Jorgensen, A. B. MacNee, and W. W. Peterson, The response of Panoramic Analyzer to CW and pulse signals, Proc. Inst. Radio Engrs. 42, 948 (1954); S. S, L. Chang, On the filter problem of the power-spectrum analyzer, Proc. Inst. Radio Engrs. 42, 1278 (1954). 
The relations between $Q$ and the sweep rate follow from the restrictions of eq (2) on minimum dwell time:

$$
\Delta \tau=\frac{\alpha Q}{2 \pi f_{0}} .
$$

Describing the instantaneous scanning rate by the expression

$$
h\left(f_{0}\right) d f_{0}=d t,
$$

an approximation to $\Delta \tau$ is obtained by integrating the scanning function between the half-power points of the filter. (To an approximation good to $12 \frac{1}{2}$ percent, even for $Q$ as low as 1 , the limits are $f_{0}$ $\pm f_{0} / 2 Q$.) Thus,

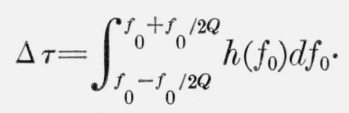

Expanding this integral into Taylor's series:

$$
\begin{aligned}
\Delta \tau \cong \theta\left(f_{0}\right)+f_{0} / 2 Q \Theta^{\prime}\left(f_{0}\right)+\left(\frac{f_{0}}{2 Q}\right)^{2} \frac{\Theta^{\prime \prime}\left(f_{0}\right)}{2}+ \\
\left(\frac{f_{0}}{2 Q}\right)^{3} \frac{\Theta^{\prime \prime \prime}\left(f_{0}\right)}{3 !}+\cdots-\Theta\left(f_{0}\right)+\frac{f_{0}}{2 Q} \Theta^{\prime}\left(f_{0}\right)- \\
\left(\frac{f_{0}}{2 Q}\right)^{2} \frac{\theta^{\prime \prime}\left(f_{0}\right)}{2}+\left(\frac{f_{0}}{2 Q}\right)^{3} \frac{\theta^{\prime \prime \prime}\left(f_{0}\right)}{3 !}+\cdots
\end{aligned}
$$

So that, to a close approximation:

$$
\Delta \tau=\frac{f_{0}}{Q} \Theta^{\prime}\left(f_{0}\right)+\frac{1}{3}\left(\frac{f_{0}}{2 Q}\right)^{3} \Theta^{\prime \prime \prime}\left(f_{0}\right)+\ldots
$$

Looking back at the original integral, evidently

$$
\theta^{\prime}=h\left(f_{0}\right)
$$

and, substituting into the series

$$
\Delta \tau=\frac{f_{0}}{Q} h\left(f_{0}\right)+\frac{1}{3}\left(\frac{f_{0}}{2 Q}\right)^{3} h^{\prime \prime}\left(f_{0}\right)+\text { higher terms. }
$$

But, from eq (3)

$$
\Delta \tau=\frac{\alpha Q}{2 \pi f_{0}}=\frac{f_{0}}{Q} h\left(f_{0}\right)+\frac{1}{3}\left(\frac{f_{0}}{2 Q}\right)^{3} h^{\prime \prime \prime}\left(f_{0}\right), \text { etc. }
$$

If $Q$ is of moderate size or higher, then, very nearly

$$
h\left(f_{0}\right)=\frac{\alpha}{2 \pi} \frac{Q^{2}}{f_{0}^{2}} .
$$

The precise way in which $Q$ depends on the total scan time, the scanning function, and the total bandwidth covered can be obtained by substituting for eq (4) in the expression

$$
h\left(f_{0}\right) d f_{0}=d t .
$$

Assigning the desired variation of $Q$ with frequency, and integrating for the total scan time $\tau$ between the band limits $f_{b}$ and $f_{t}$, we get

$$
\tau=\int_{f_{b}}^{f_{t}} \frac{\alpha}{2 \pi} \frac{Q}{2}^{2} d f_{0} .
$$

The magnitude of $Q$ can be found by solving this equation.

Usually $\tau$ is the reciprocal of the repetition rate of the scanning filter. The value of $\alpha$ is a measure of the fineness of power-level discrimination that can be achieved. (Notice that eq (5) provides a means for assigning to $\alpha$ any desired dependence upon frequency, so that transient power discrimination can be distributed at will over the frequency range.)

The functioning of a scanning filter can be compared with a parallel channel set of bandpass filters by calculating the effective number of bandpass filters that will provide the same resolution as the scanning filter. The filters may be taken to be overlapped at their half-power points, and are calculated to cover the frequency range scanned with the same power-level resolution and figure of merit as those available from the scanning filter.

For a narrow frequency differential, $d f$, in the tuning of the scanning filter, the "concentration" of filters in the range is given by

$$
d n=\frac{d f}{\Delta f},
$$

where $\Delta f$ is the width between the half-power points of the scanning filter at the instantaneous center frequency, $f_{0}$. The figure of merit, $Q$, is, of course $f_{0} / \Delta f$. Since one-half of a filter extends beyond each limit of the frequency range defined, integrating over the range gives one less than the equivalent number of parallel-channel filters that have their center tuning extending over the range, or

$$
n_{2}-1=\int_{f_{b}}^{f_{t}} \frac{d f}{\Delta f}=\int_{f_{b}}^{f_{t}} \frac{Q}{f_{0}} d f \text {. }
$$

\section{Uniform Distribution of Transient Error}

Table 1 summarizes the results of applying the foregoing discussion to calculate $Q$, the figure of merit, $k$, the scanning-rate parameter, and $n_{2}$, the equivalent number of filters overlapped at their halfpower points, in terms of the scanning time, $\tau$, the scanning bandwidth limits $f_{b}$ and $f_{t}$, and the attenuation parameter, $\alpha$. These calculations are based on uniform distribution of transient power-level discrimination, i. e., $\alpha$ is taken as a constant.

To facilitate adjustment of the response time of an analyzer to the least duration of any signal component to be observed, the tabulation also includes $\Delta \tau$ the observation time interval for any single filter.

Although the spreading due to ringing in the scanning filter is neglected, the relations shown in table 1 indicate quantitatively how the transient error and scanning characteristics affect the resolution of an 
TABLE 1. Relationships among variables describing the performance of scanning and fixed bandpass filters in which error in iransient power-level discrimination is uniformly disiributed

\begin{tabular}{|c|c|c|c|c|}
\hline Scanning rate & $\begin{array}{l}\text { Q, Figure of merit } \\
\text { for selectivity }\end{array}$ & $\begin{array}{l}\left(n_{2}-1\right), \text { Number of fil- } \\
\text { ters overlapped at half- } \\
\text { power points }\end{array}$ & $\begin{array}{c}K \text {, Scanning } \\
\text { parameter }\end{array}$ & $\begin{array}{l}\Delta \tau, \text { Observation } \\
\text { interval }\end{array}$ \\
\hline No scan $\ldots$ & $\leq \frac{2 \pi f_{0} \Delta \tau_{0}}{\alpha}$ & $\frac{2 \pi \Delta \tau_{0}\left(f_{t}-f_{b}\right)}{\alpha}$ & 0 & $\Delta \tau_{0}=\frac{\alpha \varrho}{2 \pi f_{0}}$ \\
\hline Uniform scan, $d f / d t=k_{\sim}$ & $\leq \sqrt{\frac{2 \pi \tau}{\alpha\left(f_{t}-f_{b}\right)}} f_{0}$ & $\sqrt{\frac{2 \pi \tau\left(f_{t}-f_{b}\right)}{\alpha}}$ & $\frac{f_{t}-f_{t}}{\tau}$ & $\frac{\alpha \tau}{2 \pi\left(f_{t}-f_{b}\right)}$ \\
\hline Logarithmic sean, $d f / d t=k f_{0-}-$ & $\leq \sqrt{\frac{2 \pi \tau}{\alpha \ln \left(f_{t} / f_{b}\right)}} \sqrt{f_{0}}$ & $2 \sqrt{\frac{2 \pi \tau}{\alpha \ln \left(f_{t} / f_{b}\right)}}\left(\sqrt{f_{t}}-\sqrt{f_{b}}\right)$ & $\frac{\ln \left(f_{t} / f_{b}\right)}{\tau}$ & $\frac{1}{\sqrt{f_{0}}} \sqrt{\frac{\alpha \tau}{2 \pi \ln \left(f_{t} / f_{b}\right)}}$ \\
\hline Quadratic scan, $d f / d t=k f_{0}{ }^{2}$ & $\leq \sqrt{\frac{2 \pi \tau}{\alpha\left(1 / f_{b}-1 / f_{t}\right)}}$ & $\sqrt{\frac{2 \pi \tau}{\alpha\left(1 / f_{b}-1 / f_{t}\right)}} \ln \left(\frac{f_{t}}{f_{b}}\right)$ & $\frac{1 / f_{b}-1 / f_{t}}{\tau}$ & $\frac{1}{f_{0}} \sqrt{\frac{\alpha \tau}{2 \pi\left(1 / f_{b}-1 / f_{t}\right)}}$ \\
\hline
\end{tabular}

analyzer. The spreading due to ringing can be taken care of through the power-level resolution factor $\alpha$.

As a numerical illustration of how the factors operate, consider what sort of analysis can be accomplished over the frequency range from 100 to 10,000 cps in $0.1 \mathrm{sec}$, if we are satisfied with a 10 -percent power-level error.

From a bank of parallel bandpass filters, overlapped at their half-power points, you can get meaningful results from $6.26 \times 10^{4}$ filters, with a $Q$ ranging from $6.28 \times 10^{2}$ at $100 \mathrm{cps}$ to $6.28 \times 10^{4}$ at $10,000 \mathrm{cps}$.

If you scan uniformly at 10 sweeps/sec, the effective number of illters is markedly decreased because, of course, much less response time is available for each filter; in fact each filter must now respond with 90 percent of its final indication in about $4 \mathrm{msec}$. This sort of scan is equivalent to 252 filters, overlapped at their half-power points, and the available selectivity ranges from a $Q$ of 2.52 at 100 cps to 252 at $10,000 \mathrm{cps}$.

A logarithmic scan, which allows somewhat longer dwell time at low frequencies, is equivalent to only somewhat fewer filters, 211 being the number under the conditions of the example given above. However, the $Q$ at the low-frequency limit is 12.2 , so that there has been some improvement in low-frequency resolution. This has been obtained at some loss in high-frequency selectivity; at $10,000 \mathrm{cps}$ the $Q$ is 122 .

Where constant percentage bandwidth is desired, the scan rate is proportional to the square of the frequency. A correspondingly larger part of the time of observation is devoted to the low-frequency region. The limits converge on a $Q$ of 25.2 independent of frequency, covering the range with 116 filters.

\section{Limitation by Noise}

The noise level associated with the signal provides a practical limit to the power-level resolution that can be obtained. Any further increase in resolution merely results in observing the noise, so that like signals may appear to differ.

An especially interesting expression results from considering the least power change observable to be limited by the noise present with the signal. If $S$ is the signal power and $N$ is the noise power, we take as a limit for detection

$$
\Delta S \geq N
$$

Considered as a fractional power change,

$$
\frac{\Delta S}{S+N}=\frac{W_{0}-W_{i}}{W_{0}}=1-e^{-\alpha} \geq \frac{N}{S+N} .
$$

When $S / N \gg 1, \alpha \rightarrow N / S$ and

$$
\Delta f \Delta \tau \geq \frac{1}{2 \pi}\left(\frac{S}{N}\right)^{-3 / 2}
$$

and, correspondingly,

$$
Q \leq \frac{2 \pi \Delta \tau}{T_{0}}\left(\frac{S}{N}\right)=2 \pi f_{0} \Delta \tau\left(\frac{S}{N}\right)
$$

A higher $Q$ can thus be used when there is a good signal-to-noise ratio. Equation (6) has a provocative resemblance to the Heisenberg uncertainty principle. The resemblance is real: both are derived by related mathematics, and both are based on the idea of a least discernible power change.

A special case of practical importance is the detection of a sinusoidal signal in the presence of "white noise." (Noise energy per unit bandwidth in cycles per second is constant.) For the filter calibrated at its peak response by means of a sinusoidal signal of power $W_{c}$, the same indication will be obtained with a white noise of $E_{f}$ units per cycle per second, given by

$$
E_{f}=\frac{Q W_{c}}{\pi^{2} f_{0}}
$$

This expression is obtained by integrating over the filter response. ${ }^{5}$

\footnotetext{
${ }^{5}$ D. Bierens de Haan, Nouvelles Tables D'Intégrales Définies, eq. 6, p. 47 (Edition 1867 reprinted 1939).
} 
Detection of a sinusoidal signal of power $S_{0}$ (at the center frequency to which the filter is tuned) in the presence of a noise distribution of $n_{f}$ units per cycle per second proves to require a least time interval $\Delta \tau_{0}$, which is independent of $Q$.

$$
\Delta \tau_{0} \geq \frac{\pi n_{f}}{2 S_{0}}
$$

Narrowing the filter does decrease the amount of noise passed, but it lengthens the response time by a proportionate amount.

\section{Transfer of Results to Correlation Techniques}

The foregoing discussion of selectivity and response time can be transferred directly to the design of correlation analyzers. In a paper on synchronous detectors ${ }^{6}$ Selgin derives a relationship between the

${ }^{6}$ Paul Selgin, Harmonic output of the synchronous rectifier, J. Research NBS 47, 427 (1951) RP2267. integration time of a correlation analyzer and the $Q$ of a filter exhibiting equal selectivity,

$$
Q=\frac{\pi \tau}{T}
$$

where $T$ is the period of the synchronous detector and $\tau$ is the $\mathrm{R}-\mathrm{C}$ integration time constant. This relation permits calculation of the transient error distribution in correlation analyzers from the results in this paper.

The author expresses appreciation to Chester $\mathrm{H}$. Page, who provided much-needed advice on this work, and to whom the generalized mathematical treatment deriving the scanning function from an arbitrary $Q$ is due.

Washington, July 26, 1956. 